\title{
Parameter Estimation of an Electrochemistry-based Lithium-ion Battery Model
}

\author{
Ramin Masoudi ${ }^{\mathrm{a}, *}$, Thomas Uchida ${ }^{\mathrm{b}}$, John McPhee ${ }^{\mathrm{a}}$ \\ ${ }^{a}$ Department of Systems Design Engineering, University of Waterloo, Waterloo, ON, \\ N2L 3G1, Canada \\ ${ }^{b}$ Department of Bioengineering, Stanford University, Stanford, CA 94305-5448, U.S.A.
}

\begin{abstract}
Parameters for an electrochemistry-based Lithium-ion battery model are estimated using the homotopy optimization approach. A high-fidelity model of the battery is presented based on chemical and electrical phenomena. Equations expressing the conservation of species and charge for the solid and electrolyte phases are combined with the kinetics of the electrodes to obtain a system of differential-algebraic equations (DAEs) governing the dynamic behavior of the battery. The presence of algebraic constraints in the governing dynamic equations makes the optimization problem challenging: a simulation is performed in each iteration of the optimization procedure to evaluate the objective function, and the initial conditions must be updated to satisfy the constraints as the parameter values change. The $\varepsilon$-embedding method is employed to convert the original DAEs into a singularly perturbed system of ordinary differential equations, which are then used to simulate the system efficiently. The proposed numerical procedure demonstrates excellent perfor-
\end{abstract}

\footnotetext{
*Corresponding author. Tel.: 519-888-4567; Fax: 519-746-4791.

Email addresses: rmasoudi@uwaterloo.ca (Ramin Masoudi), tkuchida@stanford.edu (Thomas Uchida), mcphee@uwaterloo.ca (John McPhee)
} 
mance in the estimation of parameters for the Lithium-ion battery model, compared to direct methods that are either unstable or incapable of converging. The obtained results and estimated parameters demonstrate the efficacy of the proposed simulation approach and homotopy optimization procedure. Keywords: battery, homotopy, Li-ion, optimization, parameter estimation, parameter identification

\section{Introduction}

Use of rechargeable batteries as electrochemical energy storage systems has gained a great deal of attention in many industrial fields. The automotive industry has benefitted substantially from battery technology, as it has enabled the manufacture of low-emission electric, hybrid electric, and plug-in hybrid electric vehicles $[1,2,3]$. Technologies for improving battery efficiency in vehicles have been of practical interest since the first electric cars were built in the mid-1800s. The optimization of automotive battery performance is facilitated by a physics-based model that represents the dynamic behavior of the battery, and can be used to predict its interaction with the vehicle and engine (e.g., in power management simulations). Additional practical aspects such as power, weight, longevity, safety, and cost define a broad research area in battery modeling and design.

Lithium-ion batteries are the most popular rechargeable battery units due to their low weight, low self-discharge rate, and high specific energy [4]. Electrochemical processes, together with thermal effects, are the key phenomena governing the dynamic behavior of batteries. Developing an accurate model of the Lithium-ion battery plays a pivotal role in control-oriented problems 
involving hybrid electric vehicles; however, high-fidelity models contain many parameters that must be estimated. Ideally, the battery model should be as detailed as possible while remaining capable of real-time performance, which is essential in the control-oriented design of automotive systems. Although more detailed models can be more accurate, the order and degree of nonlinearity of models based on fundamental physics can make them impractical for control design purposes. Spatial and temporal dependence in the governing equations of such models result in a system of partial differential equations (PDEs) that are coupled, stiff, highly nonlinear, and time-consuming to simulate.

Different types of physics-based battery modeling, from electrochemicalto circuit-based modeling schemes, have been studied in a comprehensive survey by Seaman et al. [5]. However, circuit-based models are not accurate enough in some control-oriented problems, which are essential in electric vehicles design. Estimating parameters of a detailed battery model can be challenging due to (1) a lack of physical significance for some parameters, especially in circuit-based models, and (2) difficulty in the experimental measurement of the parameters that are physically measurable. As a result, the practical design of battery models relies on parameter estimation, where unknown parameters in the governing dynamic equations are determined from experimental or simulated data. Although research on parameter estimation of Lithium-ion batteries has been primarily focused on equivalent circuitbased battery models $[6,7,8,9]$, some work has used phenomenological battery models based on electrochemistry processes [10,11]. Forman et al. [10] used a genetic algorithm and Ramadesigan et al. [11] applied Gauss-Newton 
method, a Jacobian-based scheme, for the process of nonlinear optimization in their parameter estimation efforts, minimizing an objective function that represents the difference between the model outputs and their experimentally measured values. In general, finding a global minimum in the resulting optimization problem is the primary concern when estimating parameters. In this work, we use the homotopy optimization method $[12,13]$, which is adept at finding global optima.

The remainder of this paper is organized as follows. In Section 2, we introduce the electrochemistry-based Lithium-ion battery model developed by Newman and Tiedemann [14] and Doyle et al. [15], which takes the form of PDEs. We also discuss the reduction procedure used by Dao et al. [4] to convert these PDEs into differential-algebraic equations (DAEs). The $\varepsilon$-embedding method, an efficient math-based algorithm, is introduced in Section 3 to solve the DAEs. Accordingly, we describe our procedure for numerical simulation. In Section 4, we present the homotopy optimization procedure, a global optimization scheme applied to parameter estimation in dynamic systems by Vyasarayani et al. [13, 16]. The existence of algebraic constraint equations makes the optimization process more complicated in this application, since the initial conditions must be updated at each iteration in response to the changing parameter values. We then examine the application of homotopy optimization to estimating the parameters in the battery model in Section 5. Conclusions are discussed in Section 6. 


\section{Lithium-ion battery dynamic model}

The electrochemical Lithium-ion battery model considered in this paper is derived using two main physical concepts: porous-electrode theory and concentrated solution theory $[14,15]$. As depicted in Fig. 1, the one-dimensional battery model is composed of positive and negative composite electrodes, the separator (an electrically non-conducting layer), and the electrolyte (a solvent containing a dissolved lithium salt that acts as an ionic conductor). Lithium ions travel parallel to the $x$ axis between the positive and negative

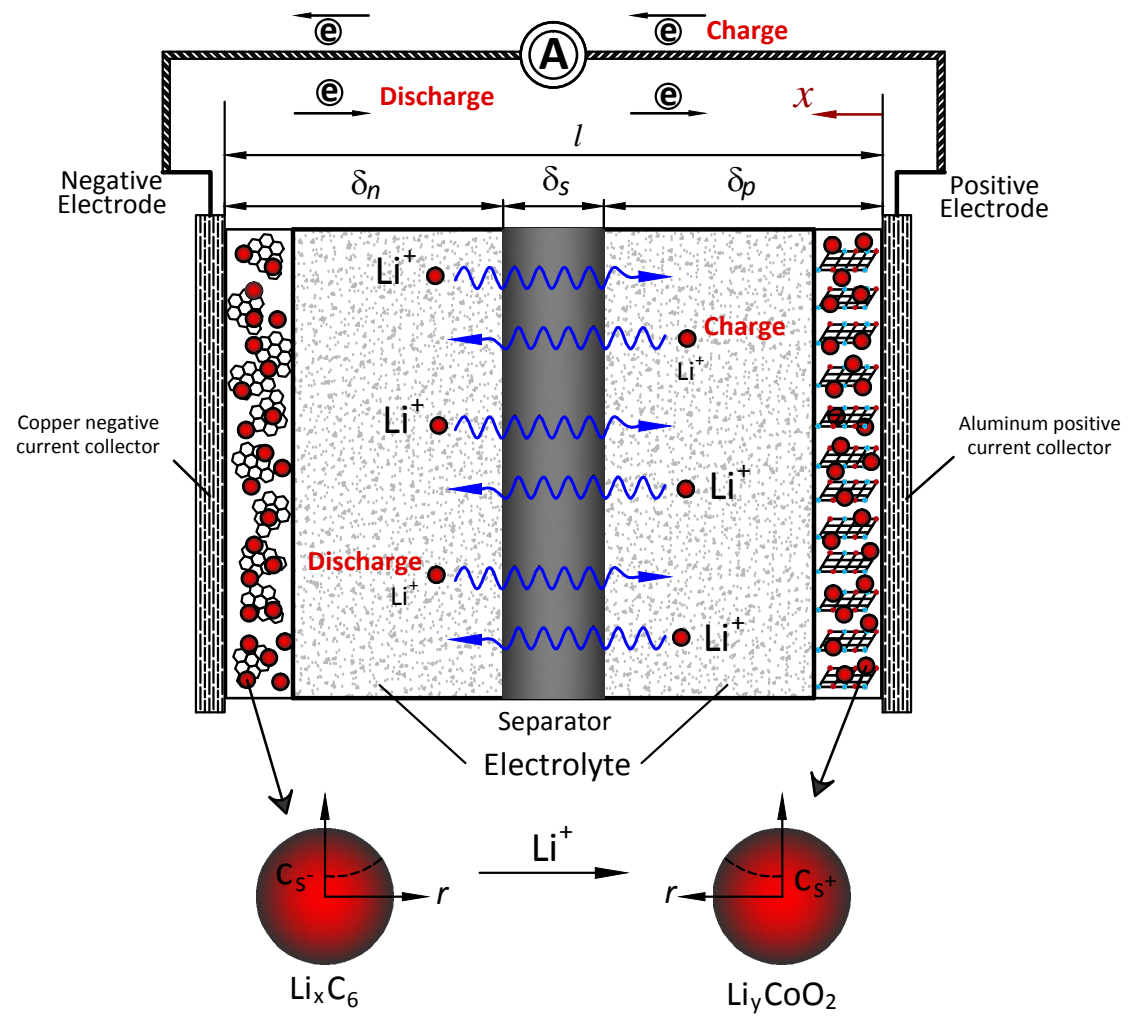

Figure 1: Schematic of a Lithium-ion battery cell.

electrodes (also called the cathode and anode) through the electrolyte by way 
of diffusion and migration. The transport of lithium ions causes electrons to flow in the external circuit [17], thereby converting stored chemical energy into electrical energy. A thorough description of the charging and discharging processes in Lithium-ion batteries has been presented by Marcicki [18] and Dao et al. [4].

The focus of this work is the estimation of parameters in the equations governing the dynamics of the battery. The physical nature of these equations is discussed briefly; we refer to full explanations of the physics-based formulations in the literature. The battery equations are derived based on five electrochemical phenomena, described below. An inventory of all the battery parameters and their numerical values used in the simulation model is provided in Table A.1.

\subsection{Species conservation for solid phase}

According to the theory of porous electrodes, the lithium in a battery cell exists in two phases: the solid phase in the electrode material and the liquid phase in the electrolyte. As lithium ions $\left(\mathrm{Li}^{+}\right)$intercalate into and out of the electrodes in the pseudo-dimension $r$ (the direction normal to the surface of the electrodes, shown in Fig. 1), the diffusion equations for the solid phase are expressed in both the $x$-direction and the pseudo-dimension $r$ [19]. Variations of lithium ion concentration in the solid phase can be expressed using Fick's laws of diffusion [15, 20], assuming $c_{s, k}=c_{s, k}(x, r, t)$ :

$$
\frac{\partial c_{s, k}}{\partial t}=\frac{D_{s, k}}{r^{2}} \frac{\partial}{\partial r}\left(r^{2} \frac{\partial c_{s, k}}{\partial r}\right)
$$

where $c_{s, k}$ and $D_{s, k}$ are, respectively, the concentration of lithium ions and the lithium ion diffusion constant coefficient associated with electrode $k$, as- 
suming $k=p$ for the positive electrode and $k=n$ for the negative electrode. Associated with Eq. (1) are the following boundary conditions:

$$
-\left.D_{s, k} \frac{\partial c_{s, k}}{\partial r}\right|_{r=0}=0 \text { and }-\left.D_{s, k} \frac{\partial c_{s, k}}{\partial r}\right|_{r=R_{s, k}}=J_{k}(x, t)
$$

as well as the following initial condition:

$$
\left.c_{s, k}\right|_{t=0}=c_{s, k, 0}
$$

where $R_{s, k}$ and $J_{k}(x, t)$ are, respectively, the constant radius of intercalation and the pore-wall flux of lithium ions associated with electrode $k$.

Subramanian et al. [21] employed polynomial approximation and volumeaverage integration to derive equations for surface and average lithium ion concentrations. Introducing a three-variable polynomial function, together with volume-average integration for the original PDE and its derivative, Dao et al. [4] obtained two equivalent ordinary differential equations (ODEs) for the average concentration of lithium ions and the corresponding flux:

$$
\begin{aligned}
\dot{\bar{c}}_{s, k}(t)+3 \frac{J_{k}(t)}{R_{s, k}} & =0 \\
\dot{\bar{q}}_{s, k}(t)+30 \frac{D_{s, k}}{R_{s, k}^{2}} \bar{q}_{s, k}(t)+\frac{45}{2} \frac{J_{k}(t)}{R_{s, k}^{2}} & =0
\end{aligned}
$$

which are coupled with an algebraic constraint equation expressing the concentration of lithium ions on the electrode surface:

$$
35 \frac{D_{s, k}}{R_{s, k}}\left(c_{s, k, \text { surf }}(t)-\bar{c}_{s, k}(t)\right)-8 D_{s, k} \bar{q}_{s, k}(t)=-J_{k}(t)
$$

where $\bar{c}_{s, k}$ is the average concentration of lithium ions on electrode $k, \bar{q}_{s, k}$ is the volume-averaged concentration of lithium ion flux associated with electrode $k$, and $c_{s, k \text {,surf }}$ is the concentration of lithium ions on the surface of electrode $k$. 


\subsection{Species conservation for electrolyte phase}

The principle of material balance is applied to the electrolyte phase to evaluate variations in the concentration of lithium ions in this salt phase, presuming a superimposed continuum for solid and electrolyte phase interactions. Accordingly, for the three distinct regions of the battery (i.e., the separator and the two porous electrodes), we have the following relation:

$$
\epsilon_{k} \frac{\partial c_{e, k}}{\partial t}=\frac{\partial}{\partial x}\left(D_{\mathrm{eff}, k} \frac{\partial c_{e, k}}{\partial x}\right)+a_{k}\left(1-t_{+}\right) J_{k}(x, t)
$$

where $k=p$ for the positive electrode, $k=s$ for the separator, and $k=n$ for the negative electrode. In Eq. (6), $\epsilon_{k}$ is the volume fraction of region $k$; $c_{e, k}=c_{e, k}(x, t)$ is the electrolyte concentration in region $k ; D_{\text {eff }, k}$ is the effective diffusion coefficient, which is calculated from a reference coefficient using

the Bruggman relation $D_{\text {eff }, k}=D \epsilon_{k}^{\text {brugg }_{k}}$ that accounts for the tortuous path that lithium ions follow through the porous media [4]; $D$ is the electrolyte diffusion coefficient; $a_{k}$ is the specific surface area of electrode $k$; $\operatorname{brugg}_{k}$ is the Bruggman power coefficient of region $k$; and $t_{+}$is the lithium ion transference number associated with the electrolyte. The boundary conditions are imposed assuming zero flux - and, therefore, zero concentration gradient - at the positive and negative current collectors:

$$
-\left.D_{\mathrm{eff}, k} \frac{\partial c_{e, p}}{\partial x}\right|_{x=0}=0 \quad \text { and } \quad-\left.D_{\mathrm{eff}, k} \frac{\partial c_{e, n}}{\partial x}\right|_{x=l}=0
$$


along with continuity of the flux and concentration of the electrolyte at the electrode-separator interface:

$$
\begin{aligned}
& \left\{\begin{array}{l}
\left.c_{e, p}\right|_{x=\delta_{p}^{-}}=\left.c_{e, s}\right|_{x=\delta_{p}^{+}} \\
-\left.D_{\mathrm{eff}, p} \frac{\partial c_{e, p}}{\partial x}\right|_{x=\delta_{p}^{-}}=-\left.D_{\mathrm{eff}, s} \frac{\partial c_{e, s}}{\partial x}\right|_{x=\delta_{p}^{+}}
\end{array}\right. \\
& \left\{\begin{array}{l}
\left.c_{e, s}\right|_{x=\left(\delta_{p}+\delta_{s}\right)^{-}}=\left.c_{e, n}\right|_{x=\left(\delta_{p}+\delta_{s}\right)^{+}} \\
-\left.D_{\mathrm{eff}, s} \frac{\partial c_{e, s}}{\partial x}\right|_{x=\left(\delta_{p}+\delta_{s}\right)^{-}}=-\left.D_{\mathrm{eff}, n} \frac{\partial c_{e, n}}{\partial x}\right|_{x=\left(\delta_{p}+\delta_{s}\right)^{+}}
\end{array}\right.
\end{aligned}
$$

In Eq. (6), the specific surface area of the electrodes can be expressed in terms of the porosity of the electrode [21]:

$$
a_{k}=\frac{3}{R_{s, k}}\left(1-\epsilon_{k}-\epsilon_{f, k}\right)
$$

where $\epsilon_{f, k}$ is the volume fraction of fillers in region $k$.

Galerkin's approach, which can be categorized as a weighted-residual formulation, uses the orthogonality of a set of basis functions (satisfying the boundary conditions of the problem) to find an approximate solution to PDEs. The Galerkin method was applied by Dao et al. [4] to discretize the PDE that governs the lithium ion concentration gradient in the electrolyte phase (Eq. (6)) to obtain a corresponding reduced-order system of ODEs. This discretization scheme is applied using sinusoidal shape functions to approximate the lithium ion concentration in the electrolyte phase as follows:

$$
\hat{c}_{e, k}(x, t)=c_{e, 0}+\sum_{j=1}^{v} \cos \left(\frac{j \pi x}{l}\right) \eta_{j}(t)
$$

where $\hat{c}_{e, k}(x, t)$ is the approximate solution for the electrolyte phase concentration, $\eta_{j}(t)$ is the $j^{\text {th }}$ generalized concentration corresponding to the $j^{\text {th }}$ 
basis function, and $v$ is the number of nodes, which must be chosen such that the dynamics of the lithium ion concentration are represented to the required degree of accuracy. The residual, which is obtained by substituting the assumed solution (Eq. (10)) into the original PDE (Eq. (6)), must be orthogonal to the set of basis functions. The ODEs, extracted using the assumed solution along with the orthogonality principle, can be formulated as follows:

$$
\dot{\boldsymbol{\eta}}=-\mathbf{A}_{s p} \boldsymbol{\eta}+\mathbf{B}_{s p}
$$

where $\boldsymbol{\eta}=\left[\eta_{1}(t), \eta_{2}(t), \cdots, \eta_{v}(t)\right]^{\mathrm{T}}$ is the vector of unknown variables representing time-dependent concentration quantities, and $\mathbf{A}_{s p}$ and $\mathbf{B}_{s p}$ are constant square matrices of dimension $v$ obtained from the orthogonality conditions [4].

\subsection{Charge conservation for solid phase}

The conservation of charge in the solid phase of the battery electrodes is dominated by Ohm's law:

$$
\sigma_{\mathrm{eff}, k} \frac{\partial^{2} \Phi_{s, k}(x, t)}{\partial x^{2}}=a_{k} F J_{k}(x, t)
$$

accompanied by the following boundary conditions at the collectors:

$$
\left\{\begin{array}{l}
-\left.\sigma_{\mathrm{eff}, p} \frac{\partial \Phi_{s, p}(x, t)}{\partial x}\right|_{x=0}=-\left.\sigma_{\mathrm{eff}, n} \frac{\partial \Phi_{s, n}(x, t)}{\partial x}\right|_{x=l}=I \\
-\left.\sigma_{\mathrm{eff}, p} \frac{\partial \Phi_{s, p}(x, t)}{\partial x}\right|_{x=\delta_{p}}=-\left.\sigma_{\mathrm{eff}, n} \frac{\partial \Phi_{s, n}(x, t)}{\partial x}\right|_{x=\delta_{p}+\delta_{s}}=0
\end{array}\right.
$$

where $F$ is the Faraday's constant, $I$ is applied current density, defined as the applied current per unit surface area of the electrode, and $\sigma_{\text {eff }, k}$ is the 
effective electrical conductivity for electrode $k$, represented in terms of the porosity of the electrode:

$$
\sigma_{\mathrm{eff}, k}=\sigma_{k}\left(1-\epsilon_{k}-\epsilon_{f, k}\right), \quad k=p, n
$$

The differential equations for conservation of charge in the solid phase can be solved analytically, assuming $J_{k}(x, t)$ is constant. For the positive electrode with $J_{p}=I / a_{p} F \delta_{p}$ constant and using the boundary condition $x=0$, the closed-form solution is as follows:

$$
\Phi_{s, p}(x, t)=-\frac{1}{2} \frac{a_{p} F J_{p}}{\sigma_{\mathrm{eff}, p}} x^{2}+\frac{I}{\sigma_{\mathrm{eff}, p}} x+f_{p}(t)
$$

In a similar process for the negative electrode using the boundary condition $x=l$, we obtain the following charge conservation equation:

$$
\Phi_{s, n}(x, t)=-\frac{1}{2} \frac{a_{n} F J_{n}}{\sigma_{\mathrm{eff}, n}} x^{2}+\frac{a_{n} F J_{n}\left(\delta_{s}+\delta_{p}\right)}{\sigma_{\mathrm{eff}, n}} x+f_{n}(t)
$$

The algebraic equations expressing the conservation of charge for the positive and negative electrodes can then be solved simultaneously with the differential equations for the battery to evaluate the unknown functions $f_{p}$ and $f_{n}$.

\subsection{Charge conservation for electrolyte phase}

To derive the electrical potential conservation equations for the electrolyte phase, Kirchhoff's and Ohm's laws are applied to represent the interaction between the solid and electrolyte phases. The charge conservation equations for the electrolyte phase can be written as follows:

$$
-\sigma_{\mathrm{eff}, k} \frac{\partial \Phi_{s, k}(x, t)}{\partial x}-\kappa_{\mathrm{eff}, k} \frac{\partial \Phi_{e, k}(x, t)}{\partial x}+\frac{2 \kappa_{\mathrm{eff}, k} R T}{F}\left(1-t_{+}\right) \frac{\partial \ln c_{e, k}}{\partial x}=I
$$


where $R$ is the universal gas constant and $T$ is absolute temperature. We also have the following boundary conditions, which reflect continuity of $\Phi_{e, k}$ :

$$
\left\{\begin{array}{l}
-\left.\kappa_{\mathrm{eff}, p} \frac{\partial \Phi_{e, p}}{\partial x}\right|_{x=0}=-\left.\kappa_{\mathrm{eff}, n} \frac{\partial \Phi_{e, n}}{\partial x}\right|_{x=l}=0 \\
-\left.\kappa_{\mathrm{eff}, p} \frac{\partial \Phi_{e, p}}{\partial x}\right|_{x=\delta_{p}^{-}}=-\left.\kappa_{\mathrm{eff}, s} \frac{\partial \Phi_{e, s}}{\partial x}\right|_{x=\delta_{p}^{+}}=-\left.\kappa_{\mathrm{eff}, n} \frac{\partial \Phi_{e, n}}{\partial x}\right|_{x=\left(\delta_{p}+\delta_{s}\right)^{+}}
\end{array}\right.
$$

where $\kappa_{\text {eff }, k}$ is the effective diffusion conductivity, which is a function of the electrolyte concentration represented by the empirical Bruggman relation:

$$
\kappa_{\mathrm{eff}, k}=\epsilon_{k}^{\mathrm{brugg}_{k}} f_{d c}\left(c_{e, k}\right)
$$

Doyle et al. [22] introduced a fourth-order polynomial function for $f_{d c}$ that accurately fits the experimental conductivity data collected for various liquid/salt/polymer systems. Parameters for a system consisting of a 2:1 volume mixture of ethylene carbonate and dimethyl carbonate at $25^{\circ} \mathrm{C}$ can be found in the literature $[21,4]$.

In a similar fashion as in Section 2.2, Galerkin's method is used to discretize the obtained PDE for the electrolyte phase potential. Given the boundary conditions of the problem (Eq. (18)), the following finite series can be used [4]:

$$
\hat{\Phi}_{e, k}(x, t)=\sum_{j=1}^{v} \cos \left(\frac{j \pi x}{l}\right) \rho_{j}(t)
$$

where $\hat{\Phi}_{e, k}(x, t)$ is the assumed solution that approximates the real solution of the PDE, and $\rho_{j}(t)$ is the $j^{\text {th }}$ generalized charge corresponding to the $j^{\text {th }}$ basis function. The residual is defined as the expression obtained upon substituting the approximate solution into the original PDE; when considered 
together with the orthogonality condition, a reduced-order system of algebraic equations is obtained due to the absence of time-differentiation terms in the PDE:

$$
\mathbf{A}_{\phi}(t) \boldsymbol{\rho}+\mathbf{B}_{\phi}(t) \boldsymbol{\eta}+\mathbf{D}_{\phi} \boldsymbol{f}_{\phi}(t)+\mathbf{E}_{\phi}=\mathbf{0}
$$

where $\mathbf{A}_{\phi}(t), \mathbf{B}_{\phi}(t), \mathbf{D}_{\phi}$, and $\mathbf{E}_{\phi}$ are coefficient matrices, $\boldsymbol{\rho}$ is the vector of generalized charges, and $\boldsymbol{f}_{\phi}(t) \triangleq\left[f_{p}(t), f_{p}(t), \ldots, f_{n}(t)\right]^{\mathrm{T}}$. Note that $\boldsymbol{\eta}$ appears in the obtained equations due to term $\ln \left(c_{e, k}\right)$ in the original PDE.

\subsection{Kinetics of electrodes}

Considering a charge-transfer resistance, the kinetics of the electrodes can be expressed using the Butler-Volmer equation [23], which relates the molar pore-wall flux to the composition of the intercalating species (i.e., the concentration of lithium ions in the electrodes and the electrolyte) and the intercalation over-potential $\left(\mu_{s, k}(x, t)\right)$ :

$$
\begin{aligned}
J_{k}(x, t)= & K_{k}\left(c_{s, k, \max }-c_{s, k, \text { surf }}\right)^{0.5}\left(c_{s, k, \text { surf }}\right)^{0.5} c_{e, k}^{0.5}(x, t) \\
& \times\left[\exp \left(\frac{0.5 F}{R T} \mu_{s, k}(x, t)\right)-\exp \left(-\frac{0.5 F}{R T} \mu_{s, k}(x, t)\right)\right]
\end{aligned}
$$

where $K_{k}$ is the intercalation/deintercalation reaction-rate constant of electrode $k$. The over-potential depends on the difference between the potentials in the solid and solution phases as well as the open-circuit potential of the intercalation electrode $U_{k}$ :

$$
\mu_{s, k}(x, t)=\left(\Phi_{s, k}(x, t)-\Phi_{e, k}(x, t)\right)-U_{k}\left(\theta_{k}(x, t)\right), \quad k=p, n
$$


Open-circuit potentials for positive $\left(\mathrm{LiCoO}_{2}\right)$ and negative $\left(\mathrm{LiC}_{6}\right)$ electrodes can be expressed as follows [4]:

$$
\begin{aligned}
& U_{p}(t)=\frac{0.531 \theta_{p}^{5}-1.507 \theta_{p}^{3}+5.839 \theta_{p}-4.875}{\theta_{p}-1.005} \\
& U_{n}(t)=-0.1 \theta_{n}+0.15+0.00778 \theta_{n}^{-1}
\end{aligned}
$$

where $\theta_{k}(x, t)=c_{s, k, \text { surf }}(x, t) / c_{s, k, \max }$. Equations (24) are reduced-order versions of the relations derived from experimental data by Doyle et al. [15].

In summary, Eqs. (4a), (4b), (5), (11), (21), (22) and (24) form a system of differential-algebraic equations that must be solved simultaneously. Note that $\Phi_{s, p}(x, t)\left(\right.$ Eq. (15)) and $\Phi_{s, n}(x, t)$ (Eq. (16)) are substituted into Eq. (17); hence, $f_{p}(t)$ and $f_{n}(t)$ appear in the final equations.

\section{Numerical simulation procedure}

The dynamic equations for the physics-based battery model are semiexplicit systems of differential-algebraic equations (DAEs):

$$
\sum:\left\{\begin{array}{l}
\dot{\boldsymbol{x}}=\boldsymbol{f}(\boldsymbol{x}(t), \boldsymbol{w}(t)) \\
\mathbf{0}=\boldsymbol{g}(\boldsymbol{x}(t), \boldsymbol{w}(t))
\end{array}\right.
$$

where $\boldsymbol{f}$ and $\boldsymbol{g}$ are nonlinear vector functions, and state vectors $\boldsymbol{x}: \mathbb{R} \rightarrow \mathbb{R}^{4+v}$ and $\boldsymbol{w}: \mathbb{R} \rightarrow \mathbb{R}^{6+v}$ are defined as follows:

$$
\begin{aligned}
\boldsymbol{x} & =\left[\bar{c}_{s, p}(t), \bar{c}_{s, n}(t), \bar{q}_{s, p}(t), \bar{q}_{s, n}(t), \boldsymbol{\eta}\right]^{\mathrm{T}} \\
\boldsymbol{w} & =\left[f_{p}(t), f_{n}(t), U_{p}(t), U_{n}(t), c_{s, p, \operatorname{surf}}(t), c_{s, n, \operatorname{surf}}(t), \boldsymbol{\rho}\right]^{\mathrm{T}}
\end{aligned}
$$

The obtained equations are highly stiff due to substantially different time scales for the concentration and electrochemical polarization dynamics involved in the model. 
The most challenging aspect of solving DAEs in the form of Eq. (25) is satisfying the algebraic constraint equations: the solution to the problem must satisfy all constraints within the specified tolerance. A solution technique that prevents the accumulation of significant constraint violations is essential in this work, as errors in the solution will affect the optimization process and, ultimately, the estimated parameters. There are several strategies for transforming high-index DAEs into ODEs. When using Baumgarte stabilization [24], for example, the constraint equations are replaced with a linear combination of the constraints and their first and second time derivatives, but it can be difficult to select the stabilization parameters. In this work, we employ a reduction method called the $\varepsilon$-embedding method $[25,26]$, which performs well in our application. In this math-based transformation procedure, a singularly perturbed system of ODEs is acquired by introducing an artificial parameter $\varepsilon>0$ into the original DAEs, Eq. (25):

$$
\sum:\left\{\begin{array}{l}
\dot{\boldsymbol{x}}=\boldsymbol{f}(\boldsymbol{x}(t), \boldsymbol{w}(t)) \\
\varepsilon \dot{\boldsymbol{w}}=\boldsymbol{g}(\boldsymbol{x}(t), \boldsymbol{w}(t))
\end{array}\right.
$$

An approximate solution is obtained for the original DAE system, with decreasing error as $\varepsilon \rightarrow 0$.

To evaluate the accuracy of the $\varepsilon$-embedding method applied to the Lithium-ion battery model, we simulate the original system (Eq. (25)) in MapleSim [27] and compare the results to those obtained when using the same model parameters in the approximate system (Eq. (27)). We use the variable-step, implicit Rosenbrock third-fourth-order Runge-Kutta integrator in MapleSim to solve the original DAE system, and a stiff multi-step integrator, ode15s, in Matlab to solve the approximate system of ODEs. Ab- 
solute and relative tolerances for the solver in MapleSim were $10^{-7}$ and for the Matlab integrator were $10^{-3}$. To validate the performance of the proposed solution scheme, we compare the results obtained for the battery cell voltage, which is one of the key outputs of the battery model:

$$
V_{\text {cell }}=\Phi_{s, p}(0, t)-\Phi_{s, n}(l, t)
$$

Results obtained using a discharge rate of 25 A for a battery consisting of 70 cells are shown in Fig. 2. Clearly, we obtain an excellent agreement

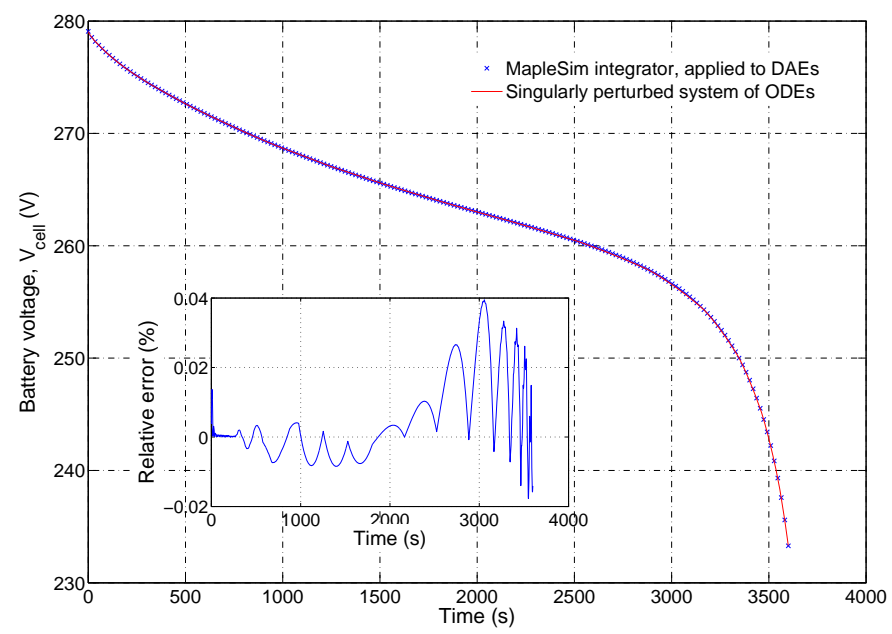

Figure 2: Comparison of discharge battery voltage obtained from the original DAE system and the approximate ODE system.

between the two models, considering a very small value of $\varepsilon=10^{-15}$. Also note that the violations of the algebraic constraints remain small throughout the duration of the simulation, as shown in Fig. 3, again demonstrating the efficacy of the proposed solution scheme. 


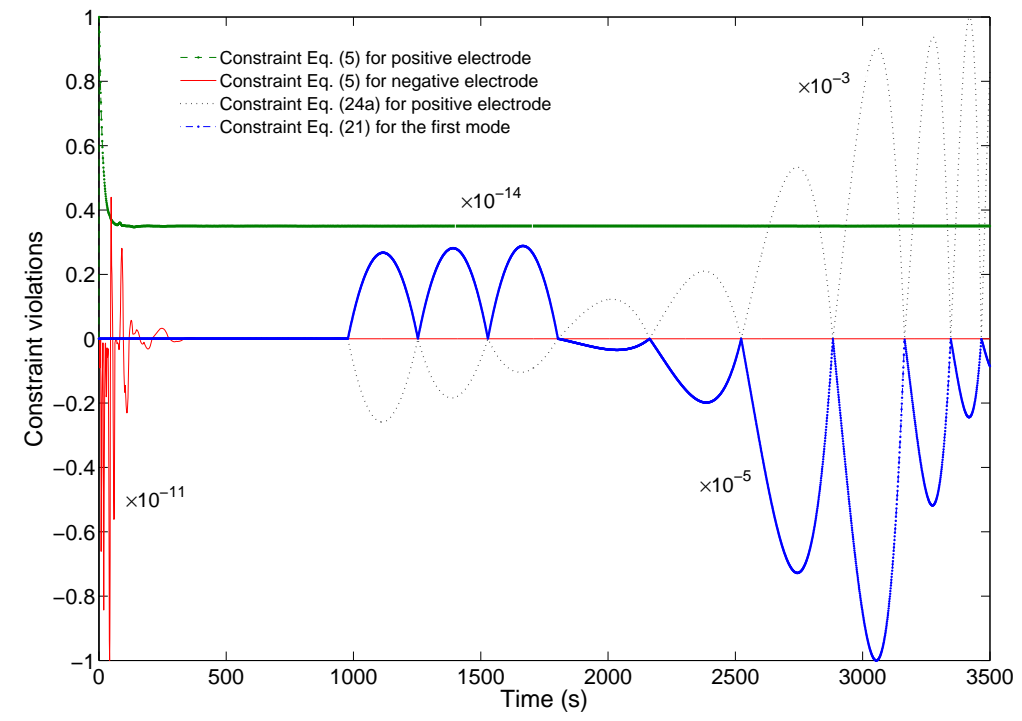

Figure 3: Normalized violations of algebraic constraint equations in the battery model (the scaling factors are shown beside each curve).

\section{Homotopy optimization method}

To improve the predictions of the battery model, the model parameters should be estimated from experimental data or a more accurate model. In this paper, we estimate model parameters using simulations of the detailed full-order model. Deterministic optimization methods are straightforward to implement for solving parameter estimation problems, but they often converge to local minima. The homotopy optimization method [12] addresses this limitation, and has been shown to be an effective technique for solving parameter estimation problems in dynamic systems $[13,16]$.

Consider the following system of ODEs:

$$
\dot{\vec{\xi}}=\mathbf{G}(\vec{\xi}, \boldsymbol{\Gamma}, t)
$$

where $\vec{\xi}$ is the vector of state variables, $\mathbf{G}$ is a vector of nonlinear functions, 
and $\boldsymbol{\Gamma}$ is the vector of parameters to be estimated. In the homotopy optimization method, we couple a vector of experimental data $\left(\vec{\xi}_{\text {exp }}\right)$ to the original system of equations using a high-gain observer [13]:

$$
\dot{\vec{\xi}}=\mathbf{G}(\vec{\xi}, \boldsymbol{\Gamma}, t)+\lambda K_{i}\left(\vec{\xi}_{\text {exp }}-\vec{\xi}\right)
$$

The homotopy parameter $\lambda$ is decreased from 1 to 0 during the optimization process, gradually reducing the effect of the homotopy coupling term. The gain $K_{i}$ is incorporated to ensure the simulation trajectory tracks the experimental data when $\lambda=1$. We then minimize the following objective function over the simulation time $T_{s}$ :

$$
\Upsilon(\boldsymbol{\Gamma})=\frac{1}{2} \sum_{j=1}^{n}\left\{\int_{0}^{T_{s}}\left(\xi_{\text {exp }}^{j}-\xi^{j}(\boldsymbol{\Gamma}, t)\right)^{2} \mathrm{~d} t\right\}
$$

where $\xi^{j}$ and $\xi_{\text {exp }}^{j}$ are the $j^{\text {th }}$ components of $\vec{\xi}$ and $\vec{\xi}_{\exp }$, respectively. The minimization procedure is performed using an iterative method such as the Gauss-Newton algorithm, which has a quadratic rate of convergence. The parameter vector is updated based on the following recurrence relation:

$$
\boldsymbol{\Gamma}^{(r+1)}=\boldsymbol{\Gamma}^{(r)}-\delta \boldsymbol{H}^{-1}\left(\boldsymbol{\Gamma}^{(r)}\right) \boldsymbol{g}^{\mathrm{T}}\left(\boldsymbol{\Gamma}^{(r)}\right)
$$

where $\delta$ denotes the step size, $\boldsymbol{g}$ is the gradient vector, and $\boldsymbol{H}$ is the Hessian of the objective function. The gradient and Hessian are computed as follows [13]:

$$
\begin{aligned}
\boldsymbol{g}(\boldsymbol{\Gamma}) & =\frac{\partial \Upsilon}{\partial \boldsymbol{\Gamma}}=-\sum_{j=1}^{n}\left\{\int_{0}^{T_{s}}\left(\xi_{\text {exp }}^{j}-\xi^{j}(\boldsymbol{\Gamma}, t)\right) \frac{\partial \xi^{j}}{\partial \boldsymbol{\Gamma}} \mathrm{d} t\right\} \\
\boldsymbol{H}(\boldsymbol{\Gamma}) & =\frac{\partial^{2} \Upsilon}{\partial \boldsymbol{\Gamma}^{2}} \approx-\sum_{j=1}^{n}\left\{\int_{0}^{T_{s}} \frac{\partial \xi^{j}}{\partial \boldsymbol{\Gamma}} \frac{\partial \xi^{j}}{\partial \boldsymbol{\Gamma}} \mathrm{d} t\right\}
\end{aligned}
$$


We begin by setting $\lambda=1$ and selecting a value for gain $K_{i}$ that is sufficiently large such that the simulated response matches the experimental data regardless of the values of the parameters $\boldsymbol{\Gamma}$. We then select an initial guess for $\boldsymbol{\Gamma}$, find parameters that minimize the objective function (Eq. (31)), decrease $\lambda$ by a specified amount, and iterate, using the optimized parameters from the previous iteration as the new initial guess. This process is continued until $\lambda=0$, whereupon the optimized parameters correspond to the original system of equations (Eq. (29)). The homotopy transformation introduced in Eq. (30) smooths the objective function, enabling a simple gradient descent algorithm to find the global minimum at each value of $\lambda$ [28].

\section{Parameter estimation of the battery model}

We wish to determine the parameters for the electrochemistry-based Lithiumion battery model that are most difficult to measure. The volume fraction of the separator regions $\left(\epsilon_{s}\right)$, the $\mathrm{Li}^{+}$transference number in the electrolyte $\left(t_{+}\right)$, the electrical conductivity of the solid phase of electrode $n\left(\sigma_{n}\right)$, and the initial electrolyte concentration in regions $s, n$, and $p\left(c_{e 0}\right)$ are estimated given results for the battery voltage obtained using a high-fidelity model. Note that the battery voltage is easily measured experimentally, so the modeling and estimation methodologies proposed in this work can be directly validated and put to practical use in future work.

The key issue in this parameter estimation application is the presence of algebraic constraints in the dynamic equations (Eq. (25)). The initial conditions play a significant role in determining the response of this highly nonlinear dynamic system, and they must be updated at each step of the 
optimization process as the parameter estimates change. We study the effect of the parameter space dimension on the performance of the homotopy optimization scheme by estimating two parameters $\left(t_{+}\right.$and $\left.c_{e 0}\right)$ and four parameters $\left(\epsilon_{s}, t_{+}, \sigma_{n}\right.$, and $\left.c_{e 0}\right)$. We use a relation for the state of charge of the battery, since this variable is of particular practical interest [4]:

$$
S O C=V_{\text {batt }}(t)-n_{\text {cell }}\left[f_{p}(t)-f_{n}(t)\right]
$$

where $V_{\text {batt }}$ is the battery voltage and $n_{\text {cell }}$ is the number of cells in the battery pack.

The experimental and simulated battery voltages for the two-parameter estimation scenario are shown in Fig. 4. Clearly, the estimated parameters

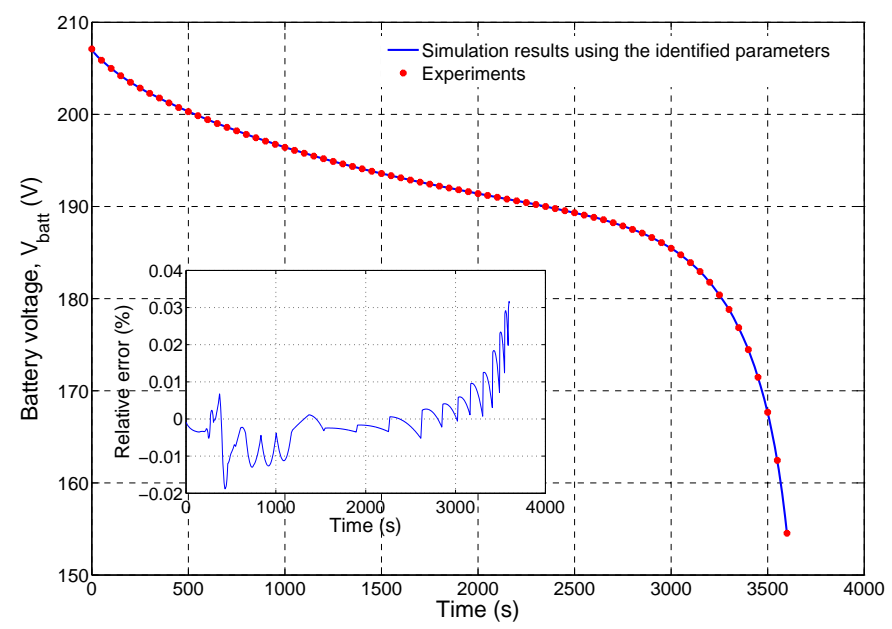

Figure 4: Time history of the battery voltage obtained experimentally and simulated with estimates for $t_{+}$and $c_{e 0}$. The battery has 51 cells, the initial state of charge is 0.9 , and the discharge rate is $28.5 \mathrm{~A}$.

result in a simulation that closely matches the experimental data. The homotopy morphing parameter and the objective function value during the 


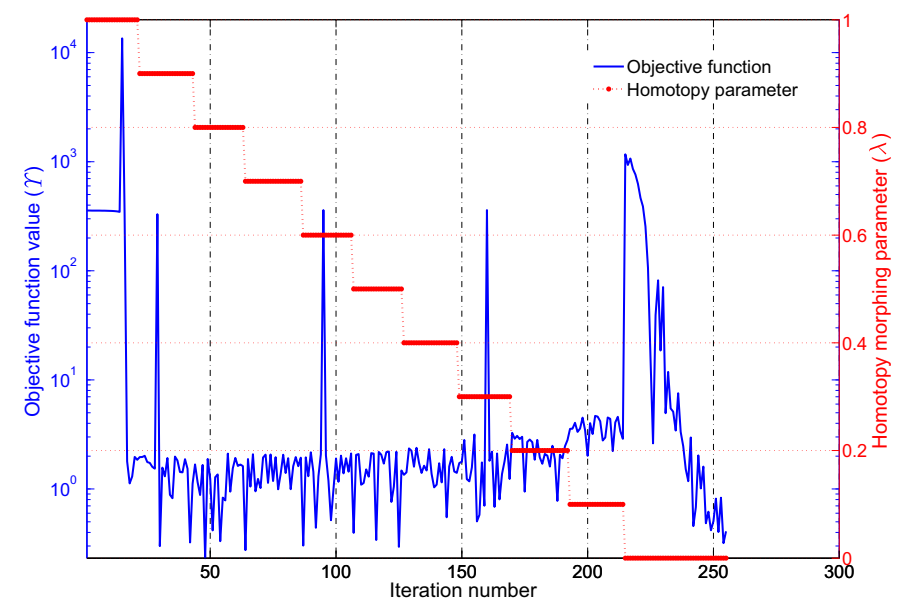

Figure 5: Homotopy morphing parameter and the corresponding objective function during the optimization process when $t_{+}$and $c_{e 0}$ are estimated. The homotopy parameter decrement is 0.1 .

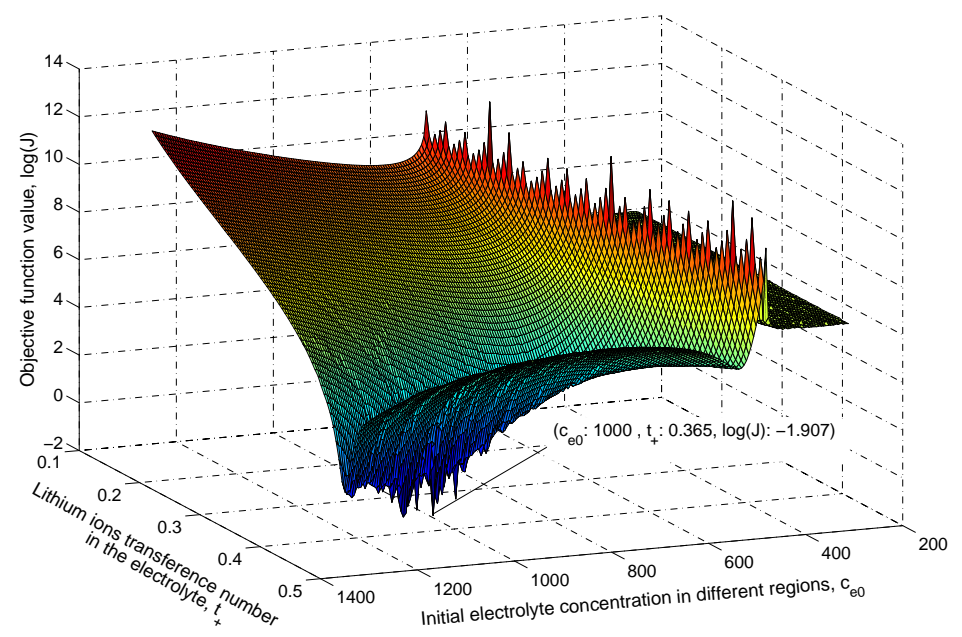

Figure 6: The value of objective function over the space of the parameters designated in the two-parameter estimation scenario.

optimization process are shown in Fig. 5; the initial guesses and final estimated parameters are provided in Table 1 . To obtained more efficient results 
during the optimization process, the number of iterations varies for different values of the homotopy parameter $\lambda$, especially for $\lambda=0$ at which the original differential equations are retrieved. The final objective function value is $0.32 \mathrm{~V}^{2}$. The value of the objective function over the space of the designated parameters exhibits several local minima, as shown in Fig. 6, demonstrating the effectiveness of the homotopy optimization approach at solving challenging minimization problems. The experimental and simulated battery voltages for the four-parameter estimation scenario are shown in Fig. 7. Even

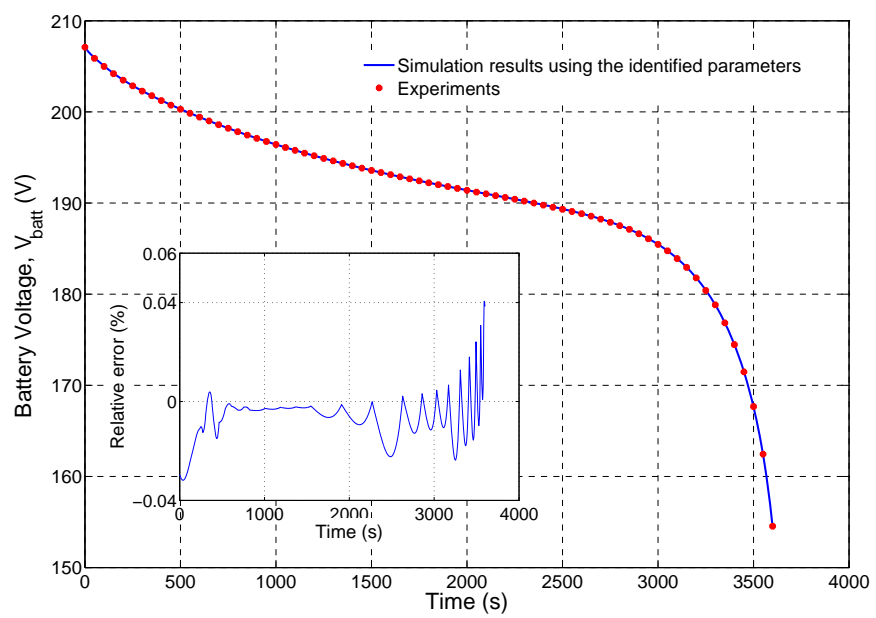

Figure 7: Time history of the battery voltage obtained experimentally and simulated with estimates for $\epsilon_{s}, t_{+}, \sigma_{n}$, and $c_{e 0}$. The battery has 51 cells, the initial state of charge is 0.9 , and the discharge rate is $28.5 \mathrm{~A}$.

\begin{tabular}{|c|c|c|c|c|}
\hline Parameter & Description & Reference value & Initial guess & Estimated value \\
\hline$t_{+}$ & $\mathrm{Li}^{+}$transference number in the electrolyte & 0.363 & 0.1 & 0.352 \\
\hline$c_{e 0}$ & Initial electrolyte concentration in regions $s, n$, and $p\left(\mathrm{~mol} \mathrm{~m}^{-3}\right)$ & 1000 & 250 & 941.52 \\
\hline
\end{tabular}

Table 1: Reference values, initial guesses, and estimated parameters for the Lithium-ion battery model in the two-parameter estimation scenario. 
in this more challenging parameter estimation scenario, the estimated parameters result in a simulation that closely matches the experimental data. The homotopy morphing parameter and the objective function value during the optimization process are shown in Fig. 8; the initial guesses and final estimated parameters are provided in Table 2. In this case, the final objec-

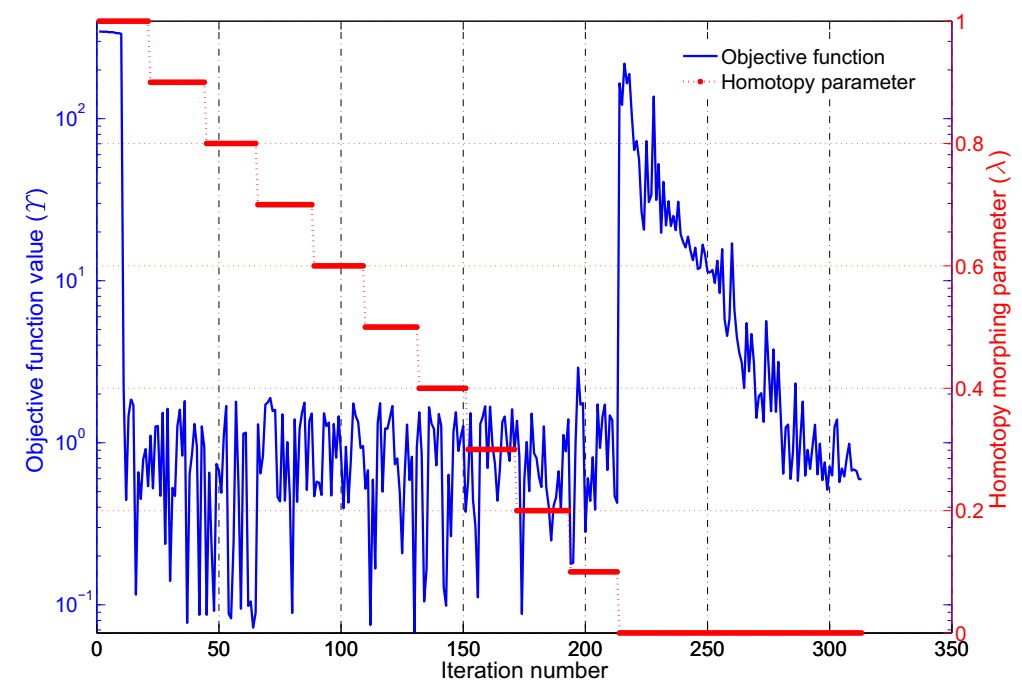

Figure 8: Homotopy morphing parameter and the corresponding objective function during the optimization process when $\epsilon_{s}, t_{+}, \sigma_{n}$, and $c_{e 0}$ are estimated. The homotopy parameter decrement is 0.1 .

\begin{tabular}{clccc}
\hline \hline Parameter & Description & Reference value & Initial guess & Estimated value \\
\hline$\epsilon_{s}$ & Volume fraction of separator region & 0.724 & 0.5 & 0.59 \\
$t_{+}$ & $\mathrm{Li}^{+}$transference number in the electrolyte & 0.363 & 0.25 & 0.27 \\
$\sigma_{n}$ & Electronic conductivity of solid phase of electrode $n\left(\mathrm{Sm}^{-1}\right)$ & 100 & 75 & 86.73 \\
$c_{e 0}$ & Initial electrolyte concentration in regions $s, n$, and $p\left(\mathrm{~mol} \mathrm{~m}^{-3}\right)$ & 1000 & 500 & 989.32 \\
\hline
\end{tabular}

Table 2: Reference values, initial guesses, and estimated parameters for the Lithium-ion battery model in the four-parameter estimation scenario.

tive function value is $0.51 V^{2}$, indicating the negative effect of increasing the 
number of estimated parameters. However, the reasonably small value for the objective function still ensures the efficacy of the optimization scheme during the parameter estimation process. It is important to note that the value of the objective function at $\lambda=0$, for which the original differential equations are retrieved, is relatively greater than its value at nonzero values of $\lambda$.

\section{Conclusions}

Parameters were estimated for an electrochemistry-based Lithium-ion battery model. The model was constructed based on porous-electrode and concentrated-solution theory, according to which the equations for the kinetics of the electrodes as well as species and charge conservations for solid and electrolyte phases were derived. The obtained governing equations for the battery consist of highly nonlinear DAEs, which are challenging to integrate due to the vastly different time scales of the physical phenomena involved in the model. We used a heuristic numerical solution scheme, the $\varepsilon$-embedding procedure, in which a singularly perturbed ODE system is obtained from the original DAEs. The transformed system was evaluated for its accuracy in satisfying the constraint violations as well as its performance in the parameter estimation algorithm.

The homotopy optimization scheme was selected for the parameter estimation process due to its reported success when applied to dynamic systems. Experimental data were coupled to the original battery model equations by a homotopy morphing parameter and a gain term, ultimately splitting the original optimization problem into a sequence of more tractable problems, as 
we showed. The performance of the numerical procedure and the efficacy of the optimization scheme were examined by studying two- and four-parameter estimation scenarios. We estimated parameters that are difficult to measure experimentally or difficult to approximate theoretically. As we demonstrated, increasing the number of estimated parameters resulted in a higher final ob-

jective function value. Determining strategies for estimating large numbers of parameters using homotopy optimization is a topic for future work.

\section{Acknowledgements}

The financial support of the NSERC/Toyota/Maplesoft Industrial Research Chair program is gratefully acknowledged.

\section{Appendix A. Parameters for the battery simulation model}


Table A.1: Nomenclature, including model parameters and numerical values.

\begin{tabular}{|c|c|c|}
\hline Symbol & Description & Numerical value (unit) \\
\hline$a_{k}$ & Specific surface area of electrode $k, k=p, n$ & $1.0\left(\mathrm{~m}^{2} \mathrm{~m}^{-3}\right)$ \\
\hline $\operatorname{brugg}_{k}$ & Bruggman power coefficient of region $k, k=p, n$ & 1.5 \\
\hline$\hat{c}_{e, k}$ & Approximate solution for the electrolyte phase concentration & $\left(\mathrm{mol} \mathrm{m}^{-3}\right)$ \\
\hline$c_{e, k}$ & Electrolyte concentration in region $k, k=p, n, s$ & $\left(\mathrm{~mol} \mathrm{~m}^{-3}\right)$ \\
\hline$c_{e, k, 0}$ & Initial electrolyte concentration in region $k, k=p, n, s$ & $\left(\mathrm{~mol} \mathrm{~m}^{-3}\right)$ \\
\hline$c_{s, k}$ & Concentration of lithium ions associated with electrode $k, k=p, n$ & $\left(\mathrm{~mol} \mathrm{~m}^{-3}\right)$ \\
\hline$c_{s, k, 0}$ & Initial concentration of lithium ions associated with electrode $k, k=p, n$ & $\left(\mathrm{~mol} \mathrm{~m}^{-3}\right)$ \\
\hline $\bar{c}_{s, k}$ & Average concentration of lithium ions on electrode $k, k=p, n$ & $\left(\mathrm{~mol} \mathrm{~m}^{-3}\right)$ \\
\hline$c_{s, k, \text { surf }}$ & Concentration of lithium ions on the surface of electrode $k, k=p, n$ & $\left(\mathrm{~mol} \mathrm{~m}^{-3}\right)$ \\
\hline$D$ & Electrolyte diffusion coefficient & $7.5 \times 10^{-11}\left(\mathrm{~m}^{2} \mathrm{~s}\right)$ \\
\hline$D_{s, k}$ & Lithium ion diffusion coefficient associated with electrode $k, k=p, n$ & $1.0 \times 10^{-14}, 3.9 \times 10^{-14}\left(\mathrm{~m}^{2} \mathrm{~s}\right)$ \\
\hline F & Faraday's constant & $96487\left(\mathrm{C} \mathrm{mol}^{-1}\right)$ \\
\hline$I$ & Applied current density & $\left(\mathrm{A} \mathrm{mol}^{-2}\right)$ \\
\hline$J_{k}(x, t)$ & Pore-wall flux of lithium ions associated with electrode $k, k=p, n$ & $\left(\mathrm{molm}^{-2} \mathrm{~s}\right)$ \\
\hline$K_{k}$ & Intercalation/deintercalation reaction-rate constant of electrode $k, k=p, n$ & $2.33 \times 10^{-11}, 5.03 \times 10^{-11}\left(\mathrm{~mol}\left(\mathrm{~mol} \mathrm{~m}^{-3}\right)^{-1.5}\right)$ \\
\hline$l$ & Total thickness of cathode-separator-anode & $(\mathrm{m})$ \\
\hline$n$ & Negative electrode & \\
\hline$p$ & Positive electrode & \\
\hline $\bar{q}_{s, k}$ & Volume-averaged concentration of lithium ion flux associated with electrode $k, k=p, n$ & $\left(\mathrm{~mol} \mathrm{~m}^{-3} \mathrm{~s}^{-1}\right)$ \\
\hline$r$ & Radial coordinate & $(\mathrm{m})$ \\
\hline$R$ & Universal gas constant & 8.314 \\
\hline$R_{s, k}$ & Radius of intercalation of lithium ions associated with electrode $k, k=p, n$ & $1 / 500000(\mathrm{~m})$ \\
\hline$s$ & Separator & \\
\hline$t_{+}$ & Lithium ion transference number associated with the electrolyte & 0.363 \\
\hline$T$ & Absolute temperature & $298.15(\mathrm{~K})$ \\
\hline$U_{k}$ & Open-circuit potential of electrode $k, k=p, n$ & $(\mathrm{~V})$ \\
\hline$x$ & Spatial coordinate & $(\mathrm{m})$ \\
\hline$\delta_{k}$ & Thickness of region $k, k=p, n, s$ & $1 / 12500,11 / 125000,1 / 40000(\mathrm{~m})$ \\
\hline$\epsilon_{k}$ & Volume fraction of region $k, k=p, n, s$ & $0.385,0.485,0.724$ \\
\hline$\epsilon_{f, k}$ & Volume fraction of fillers in region $k=p, n$ & $0.25 \times 10^{-1}, 0.326 \times 10^{-1}$ \\
\hline$\eta_{j}(t)$ & $j^{\text {th }}$ generalized concentration corresponding to the $j^{\text {th }}$ basis function & $\left(\mathrm{mol} \mathrm{m}^{-3}\right)$ \\
\hline$\theta_{k}(x, t)$ & Dimensionless concentration of lithium ions in the intercalation particle of electrode $k, k=p, n$ & \\
\hline$v$ & Number of nodes for Galerkin's approximation & \\
\hline$\sigma_{\text {eff }, k}$ & Effective electrical conductivity for electrode $k, k=p, n$ & $100\left(\mathrm{~S} \mathrm{~m}^{-1}\right)$ \\
\hline$\rho_{j}(t)$ & $j^{\text {th }}$ generalized charge corresponding to the $j^{\text {th }}$ basis function & (A) \\
\hline$\Phi_{e, k}$ & Electrolyte-phase potential in region $k, k=p, n$ & $(\mathrm{~V})$ \\
\hline$\hat{\Phi}_{e, k}$ & Assumed solution for electrolyte-phase potential & $(\mathrm{V})$ \\
\hline$\Phi_{s, k}$ & Solid-phase potential of electrode $k, k=p, n$ & $(\mathrm{~V})$ \\
\hline
\end{tabular}


[1] C. Fellner, J. Newman, High-power batteries for use in hybrid vehicles, J. Power Sources 85 (2) (2000) 229-236. doi:10.1016/S0378-7753(99)003444 .

[2] B. Kennedy, D. Patterson, S. Camilleri, Use of lithium-ion batteries in electric vehicles, J. Power Sources 90 (2) (2000) 156-162. doi:10.1016/S0378-7753(00)00402-X.

[3] J. Axsen, A. Burke, K. Kurani, Batteries for plug-in hybrid electric vehicles (PHEVs): Goals and the state of technology circa 2008, Tech. Rep. UCD-ITS-RR-08-14, Institute of Transportation Studies, University of California Davis (2008).

[4] T.-S. Dao, C. P. Vyasarayani, J. McPhee, Simplification and order reduction of lithium-ion battery model based on porouselectrode theory, J. Power Sources 198 (1) (2012) 329-337. doi:10.1016/j.jpowsour.2011.09.034.

[5] A. Seaman, T.-S. Dao, J. McPhee, A survey of mathematics-based equivalent-circuit and electrochemical battery models for hybrid and electric vehicle simulation, J. Power Sources 256 (2014) 410-423. doi:10.1016/j.jpowsour.2014.01.057.

[6] Y. Hu, S. Yurkovich, Y. Guezennec, B. Yurkovich, A technique for dynamic battery model identification in automotive applications using linear parameter varying structures, Control. Eng. Pract. 17 (10) (2009) 1190-1201. doi:10.1016/j.conengprac.2009.05.002. 
[7] C. Zhang, J. Liu, S. Sharkh, C. Zhang, Identification of dynamic model parameters for lithium-ion batteries used in hybrid electric vehicles, in: Int. Symp. on Electr. Veh. (ISEV) (2010), Beijing, China, 2010.

[8] X. Tang, X. Mao, J. Lin, B. Koch, Li-ion battery parameter estimation for state of charge, in: Am. Control. Conf. (ACC), IEEE, San Francisco, CA, USA, 2011, pp. 941-946. doi:10.1109/ACC.2011.5990963.

[9] W.-J. Yang, D.-H. Yu, Y.-B. Kim, Parameter estimation of lithium-ion batteries and noise reduction using an h-infinity filter, J. of Mech. Sci. and Technol. 27 (1) (2013) 247-256. doi:10.1007/s12206-012-1203-z.

[10] J. C. Forman, S. J. Moura, J. L. Stein, H. K. Fathy, Genetic parameter identification of the Doyle-Fuller-Newman model from experimental cycling of a $\mathrm{LiFePO}_{4}$ battery, in: Am. Control. Conf. (ACC), IEEE, San Francisco, CA, USA, 2011, pp. 362-369. doi:10.1109/ACC.2011.5991183.

[11] V. Ramadesigan, K. Chen, N. A. Burns, V. Boovaragavan, R. D. Braatz, V. R. Subramanian, Parameter estimation and capacity fade analysis of lithium-ion batteries using reformulated models, J. Electrochem. Soc. 158 (9) (2011) A1048-A1054. doi:10.1149/1.3609926.

[12] D. M. Dunlavy, D. P. O'Leary, Homotopy optimization methods for global optimization, Tech. Rep. SAND2005-7495, Sandia National Laboratories (2005).

[13] C. P. Vyasarayani, T. Uchida, A. Carvalho, J. McPhee, Parameter identification in dynamic systems using the homotopy opti- 
mization approach, Multibody Syst. Dyn. 26 (4) (2011) 411-424. doi:10.1007/s11044-011-9260-0.

[14] J. Newman, W. Tiedemann, Porous-electrode theory with battery applications, AIChE J. 21 (1) (1975) 25-41. doi:10.1002/aic.690210103.

[15] M. Doyle, T. F. Fuller, J. Newman, Modeling of galvanostatic charge and discharge of the lithium/polymer/insertion cell, J. Electrochem. Soc. 140 (6) (1993) 1526-1533. doi:10.1149/1.2221597.

[16] C. P. Vyasarayani, T. Uchida, J. McPhee, Nonlinear parameter identification in multibody systems using homotopy continuation, ASME J. Comput. Nonlinear Dyn. 7 (1) (2011) 011012. doi:10.1115/1.4004885.

[17] W. Fang, O. J. Kwon, C.-Y. Wang, Electrochemical-thermal modeling of automotive Li-ion batteries and experimental validation using a three-electrode cell, Int. J. Energy Res. 34 (2) (2010) 107-115. doi:10.1002/er.1652.

[18] J. M. Marcicki, Modeling, parametrization, and diagnostics for lithiumion batteries with automotive applications, Ph.D. thesis, The Ohio State University (2012).

[19] V. Ramadesigan, V. Boovaragavan, J. J. Carl Pirkle, V. R. Subramanian, Efficient reformulation of solid-phase diffusion in physics-based lithium-ion battery models, J. Electrochem. Soc. 157 (7) (2010) A854A860. doi:10.1149/1.3425622.

[20] T. F. Fuller, M. Doyle, J. Newman, Simulation and optimization of the 
dual lithium ion insertion cell, J. Electrochem. Soc. 141 (1) (1994) 1-10. doi:10.1149/1.2054684.

[21] V. R. Subramanian, V. Boovaragavan, V. Ramadesigan, M. Arabandi, Mathematical model reformulation for lithium-ion battery simulations: galvanostatic boundary conditions, J. Electrochem. Soc. 156 (4) (2009) A260-A271. doi:10.1149/1.3065083.

[22] M. Doyle, J. Newman, A. S. Gozdz, C. N. Schmutz, J.-M. Tarascon, Comparison of modeling predictions with experimental data from plastic lithium ion cells, J. Electrochem. Soc. 143 (6) (1996) 1890-1903. doi:10.1149/1.1836921.

[23] J. Newman, K. E. Thomas-Alyea, Electrochemical Systems, 3rd Edition, Wiley, 2004.

[24] J. Baumgarte, Stabilization of constraints and integrals of motion in dynamical systems, Comput. Methods Appl. Mech. Eng. 1 (1) (1972) 1-16. doi:10.1016/0045-7825(72)90018-7.

[25] K. Mohaghegh, R. Pulch, M. Striebel, E. Maten, Model order reduction for semi-explicit systems of differential algebraic equations, in: Proceedings 6th Vienna International Conference on Mathematical Modeling, Vienna, Austria, 2009, pp. 1256-1265.

[26] K. Mohaghegh, R. Pulch, J. ter Maten, Model order reduction using singularly perturbed systems, Tech. Rep. BUW-AMNA-OPAP 10/01, Bergische Universität Wuppertal (2010). 
[27] Maplesoft, MapleSim model gallery: Application examples created in MapleSim, "Lithium-ion battery pack" (accessed October, 2014).

[28] C. P. Vyasarayani, T. Uchida, J. McPhee, Single-shooting homotopy method for parameter identification in dynamical systems, Phys. Rev. E 85 (3) (2012) 036201. doi:10.1103/PhysRevE.85.036201. 